\title{
Correlations of Clusters of Non-Convulsive Seizure and Magnetic Resonance Imaging in a Case With GAD65- Positive Autoimmune Limbic Encephalitis
}

\author{
Rachael Gardner ${ }^{\mathrm{a}}$, Rajesh Rangaswamy ${ }^{\mathrm{b}}$, Yen-Yi Peng ${ }^{\mathrm{a}, \mathrm{c}}$
}

\begin{abstract}
With the increased availability of laboratory tests, glutamic acid decarboxylase (GAD) antibody-positive limbic encephalitis has become an emerging diagnosis. The myriad symptoms of limbic encephalitis make the diagnosis challenging. Symptoms range from seizures, memory loss, dementia, confusion, to psychosis. We present a case of a 21-year-old female with GAD65 antibody-positive limbic encephalitis. The case is unique because the clinical course suggests that nonconvulsive seizures are the major cause of this patient's clinical manifestations. The following is the thesis: systemic autoimmune disease, associated with the GAD65 antibody, gives rise to seizures, in particular, non-convulsive seizures. Temporal lobes happen to be the most susceptible sites to develop seizures. The greater part of these seizures can be non-convulsive and hard to recognize without electroencephalogram (EEG) monitoring. The variable symptoms mirror the severity and locations of these seizures. The magnetic resonance imaging (MRI) signal abnormities in the bilateral hippocampus, fornix, and mammillary body correlate with the density of these seizures in the similar manner, which suggests it is secondary to post-ictal edema.
\end{abstract}

Keywords: Autoimmune; Limbic encephalitis; GAD65 antibody; Non-convulsive seizure; Hippocampus; Fornix; Mammillary body; Electroencephalogram; Magnetic resonance imaging

\section{Introduction}

Limbic encephalitis is believed to be a disorder affecting the medial temporal lobe of the brain. It could be either a paraneoplastic or autoimmune (non-paraneoplastic) origin. The most

Manuscript accepted for publication June 20, 2016

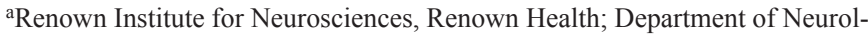
ogy, University of Nevada, Reno, NV, USA

bRadiology Department, Renown Institute for Neurosciences, Renown Health, Reno, NV, USA

${ }^{\mathrm{c} C}$ Corresponding Author: Yen-Yi Peng, Renown Institute for Neuroscience, Renown Health; Department of Neurology, University of Nevada, Reno, NV, USA. Email: ypeng@renown.org

doi: http://dx.doi.org/10.14740/jocmr2624w common non-paraneoplastic antibodies associated with limbic encephalitis are voltage-gated potassium channel antibodies (VGKC), N-methyl-D-aspartate (NMDA) receptor antibodies, and glutamic acid decarboxylase (GAD) receptor antibodies $[1,2]$.

The myriad symptoms of limbic encephalitis make correct diagnosis challenging. Symptoms of limbic encephalitis could range from seizures, memory loss, dementia, confusion, to psychosis. Furthermore, besides limbic encephalitis, GAD antibody is reported to be associated with intractable seizures with focus from frontal lobes, cerebellar ataxia, palatal myoclonus, stiff person syndrome and type I diabetic mellitus [2, 3].

GAD is the major enzyme in the synthesis of gammaamino butyric acid (GABA) which is a potent inhibitory neurotransmitter and a critical component of neurophysiologic function [3]. GAD involves two major protein isoforms that catalyze GABA synthesis. One isoform has a molecular size of $65 \mathrm{kDa}$ and is termed GAD65, while the second one, of $67 \mathrm{kDa}$ size, is termed GAD67 [3]. It is proposed that these GAD65 antibodies reduce the synthesis of GABA or interfere with exocytosis of GABA in the nervous system [4]. GAD65 antibodies act like GABA antagonists and could produce seizures $[5,6]$.

We present a case of a 21-year-old female with limbic encephalitis associated with the GAD65 antibody. The case is unique because the clinical course suggests that the non-convulsive seizures are the major cause of this patient's clinical manifestations and magnetic resonance imaging (MRI) signal abnormalities.

\section{Case Report}

We present a case of a 21-year-old female, who started having complex partial seizures 1 week prior to first hospital admission. The patient started having episodes of starring. She was exhausted, lightheaded and nauseous. She felt nauseous about half an hour after waking-up on a daily basis.

During the first admission, electroencephalogram (EEG) confirmed independent seizures from bilateral temporal areas. Anti-seizure medication was prescribed, clinical conditions improved and she was discharged. Initial MRI of the brain showed a slight abnormal increase of T2 signal intensity in the bilateral hippocampus, amygdala, fornix, and mammillary 

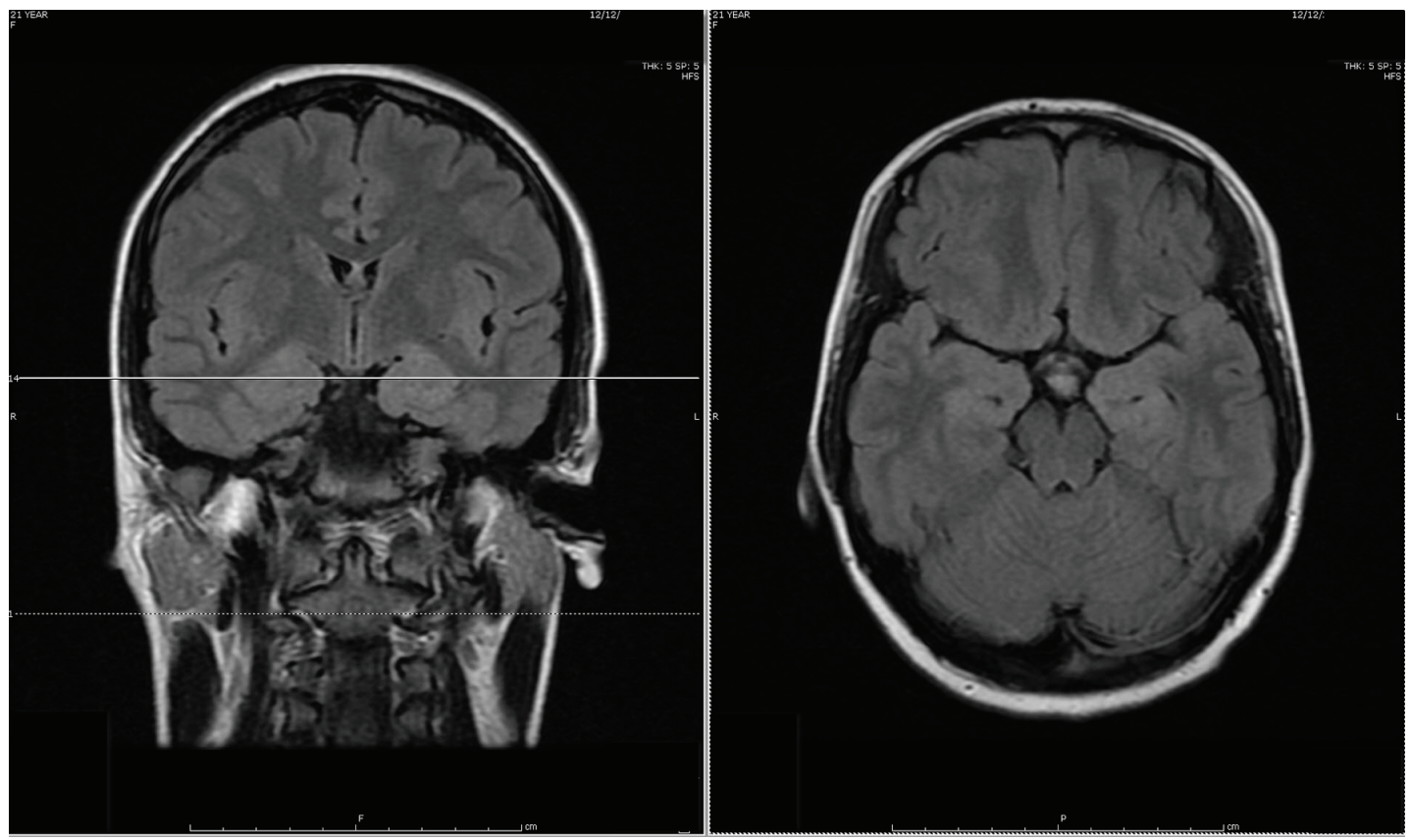

Figure 1. Initial MRI of brain showed subtle increased signal intensity on coronal and axial FLAIR MR sequences in the bilateral hippocampus, amygdala, fornix and mammillary body.

body (Fig. 1).

Despite the seizure medicine, the patient's memory continued to deteriorate. The follow-up MRI of the brain showed significant stepwise interval increase in the extent of T2 signal intensity in the bilateral hippocampus, amygdala, fornix, and mammillary body on fluid-attenuated inversion recovery
(FLAIR) sequences (Figs. 2 and 3) [7]. MRI depicted in Figs. 2 and 3 was 1 month apart.

The differential diagnosis of these MRI findings included post-ictal edema, autoimmune limbic encephalitis, paraneoplastic limbic encephalitis, and herpes encephalitis. The other remote differential diagnosis included bilateral glioma and

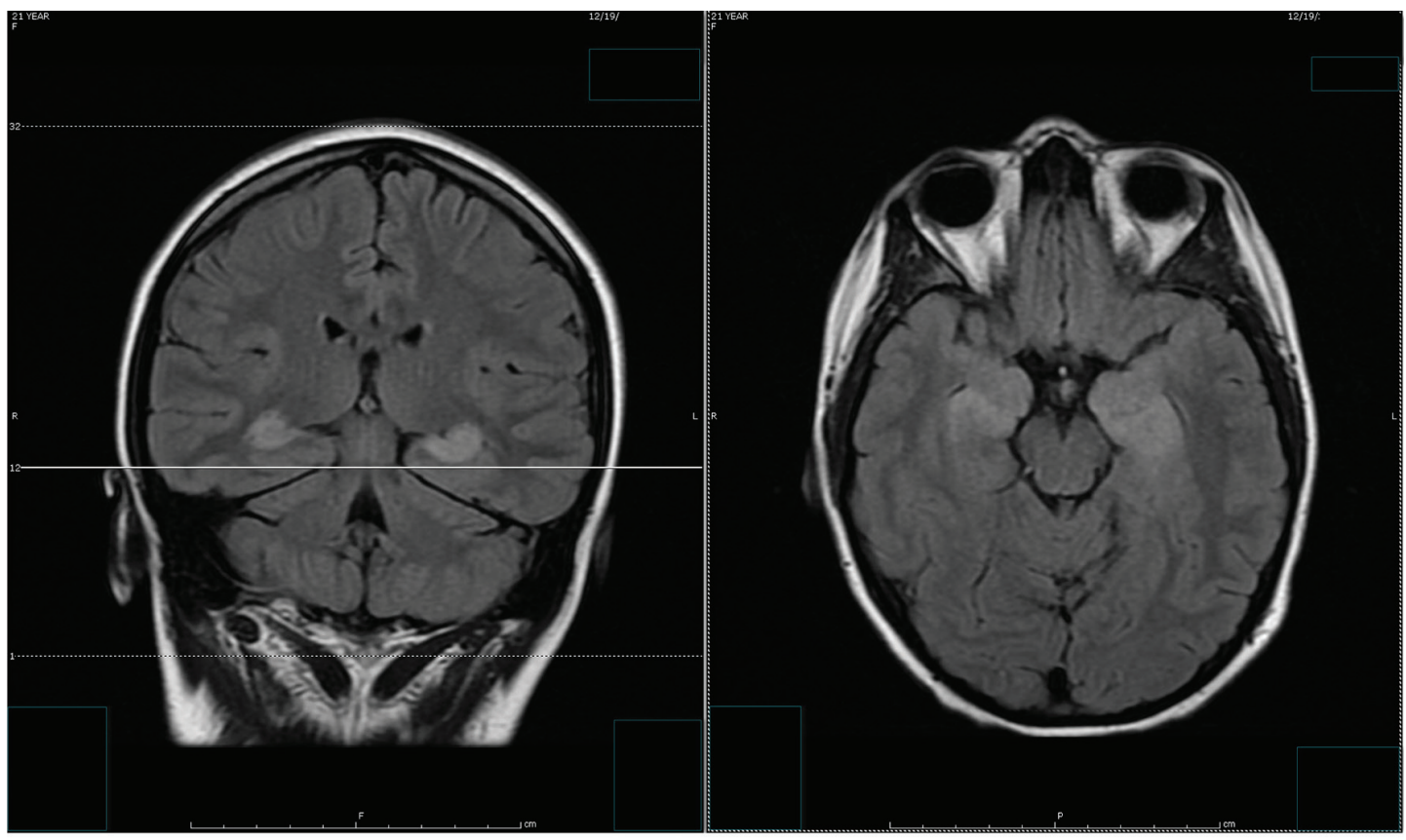

Figure 2. The follow-up MRI of brain showed interval increase in the extent of T2 signal intensity in the bilateral hippocampus, amygdala, fornix and mammillary body on fluid-attenuated inversion recovery (FLAIR) sequences. MRI obtained in Figure 1 and Figure 2 was 1 week apart. 


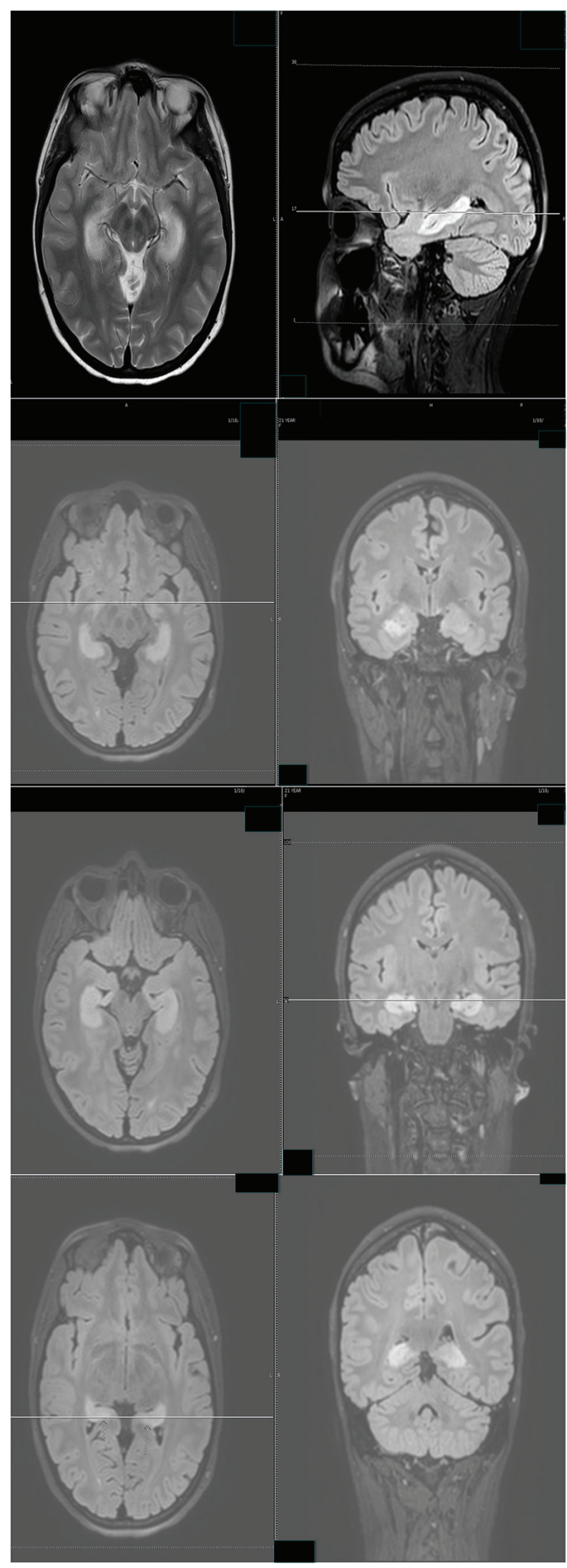

Figure 3. The follow-up MRI of brain showed significant interval increase in the extent of T2 signal intensity in the bilateral hippocampus, amygdala, fornix and mammillary body on fluid-attenuated inversion recovery (FLAIR) sequences. MRI obtained in Figure 2 and Figure 3 was 1 month apart. gliomatosis cerebri. The possibility of a tumor was considered unlikely due to the symmetric exclusive involvement of limbic system without any associated temporal lobe involvement.

Approximately 2 months after onset, the patient was readmitted. The patient's short-term memory was severely impaired. She repetitively asked the same question, which was answered a couple of minutes prior, on the first day of admission. Prolonged video EEG documented the clusters of nonconvulsive seizures (Figs. 4 and 5).

On the first day of video EEG monitoring, every 15 - 30 min, the patient had one electrical seizure. These electrical seizures started with the sudden appearance of repetitive epileptiform sharp waves over the left hemisphere, which evolved in amplitude and frequency over the left hemisphere. These epileptiform discharges did not propagate to the right hemisphere in the majority of seizures. The electrical seizures lasted around $20-30$ s each (Figs. 4 and 5). These non-convulsing seizures were difficult to recognize without EEG monitoring. In fact, the patient was able to answer simple questions during the seizures and did not show any major motor manifestations. Non-convulsive seizures continued in sleep (Figs. 4 and 5). Between seizures, the EEG background was well preserved (Fig. 6). Anti-seizure medicine was increased and intravenous solumedrol was initiated.

These non-convulsive seizure clusters, amnesia and MRI signal abnormality improved after aggressive anti-seizure medication management and intravenous solumedrol (Fig. 7). The patient was discharged with anti-seizure medicines and oral steroids.

No tumor was found on the whole body CT scan $[8,9]$. Routine complete blood cell (CBC) count, basic metabolic chemistry and routine $\mathrm{CSF}$ results were within normal range: sedimentation rate (Westergren) $6 \mathrm{~mm} / \mathrm{h}, \mathrm{CSF}$ WBC 9 cells/ $\mu \mathrm{L}, \mathrm{RBC}<1$ cells $/ \mu \mathrm{L}$, glucose $60 \mathrm{mg} / \mathrm{dL}$, total protein $35 \mathrm{mg} /$ $\mathrm{dL}$, and $\mathrm{IgG} \mathrm{CSF} 4 \mathrm{mg} / \mathrm{dL}$ (reference range $0-6 \mathrm{mg} / \mathrm{dL}$ ).

Serum GAD65 Ab was more than $250 \mathrm{IU} / \mathrm{mL}$ (reference range 0.0 - $5.0 \mathrm{IU} / \mathrm{mL}$ ) and CSF GAD65 Ab assay was 53.0 $\mathrm{nmol} / \mathrm{L}$ (the reference range $\leq 0.02 \mathrm{nmol} / \mathrm{L}$ ). The other antibodies in the Encephalopathy-Autoimmune Panel of Mayo Clinic Laboratories were negative including NMDA-R Ab CBA, VGKC-complex Ab IPA, GABA-B-R Ab CBA, AMPA-R Ab CBA, ANNA-1 (anti-neuronal nuclear Ab, type 1), ANNA-2 (anti-neuronal nuclear Ab, type 2), ANNA-3 (antineuronal nuclear Ab, type 3), AGNA-1 (anti-glial nuclear Ab, type 1), PCA-1 (Purkinje cell cytoplasmic Ab type 1), PCA-2 (Purkinje cell cytoplasmic Ab type 2), PCA-Tr (Purkinje cell cytoplasmic Ab type Tr), amphiphysin Ab, and CRMP-5-IgG negative titer $<1: 2$ (collapsin response mediator protein 5) [8].

Three months after onset, the patient was re-admitted. In this admission, the patient's non-convulsive seizures and amnesia reappeared during a transition of seizure medicines. Intravenous immunoglobulin (IVIG) and intravenous solumedrol were administered in this admission. Both non-convulsive seizures and amnesia improved.

Weeks afterward, the patient was re-admitted because of a skin rash assumed to be secondary to the seizure medicine. Both amnesia and non-convulsive seizures were again prominent on the first day. Amnesia and non-convulsive seizures improved with modification of anti-seizure medicine and in- 


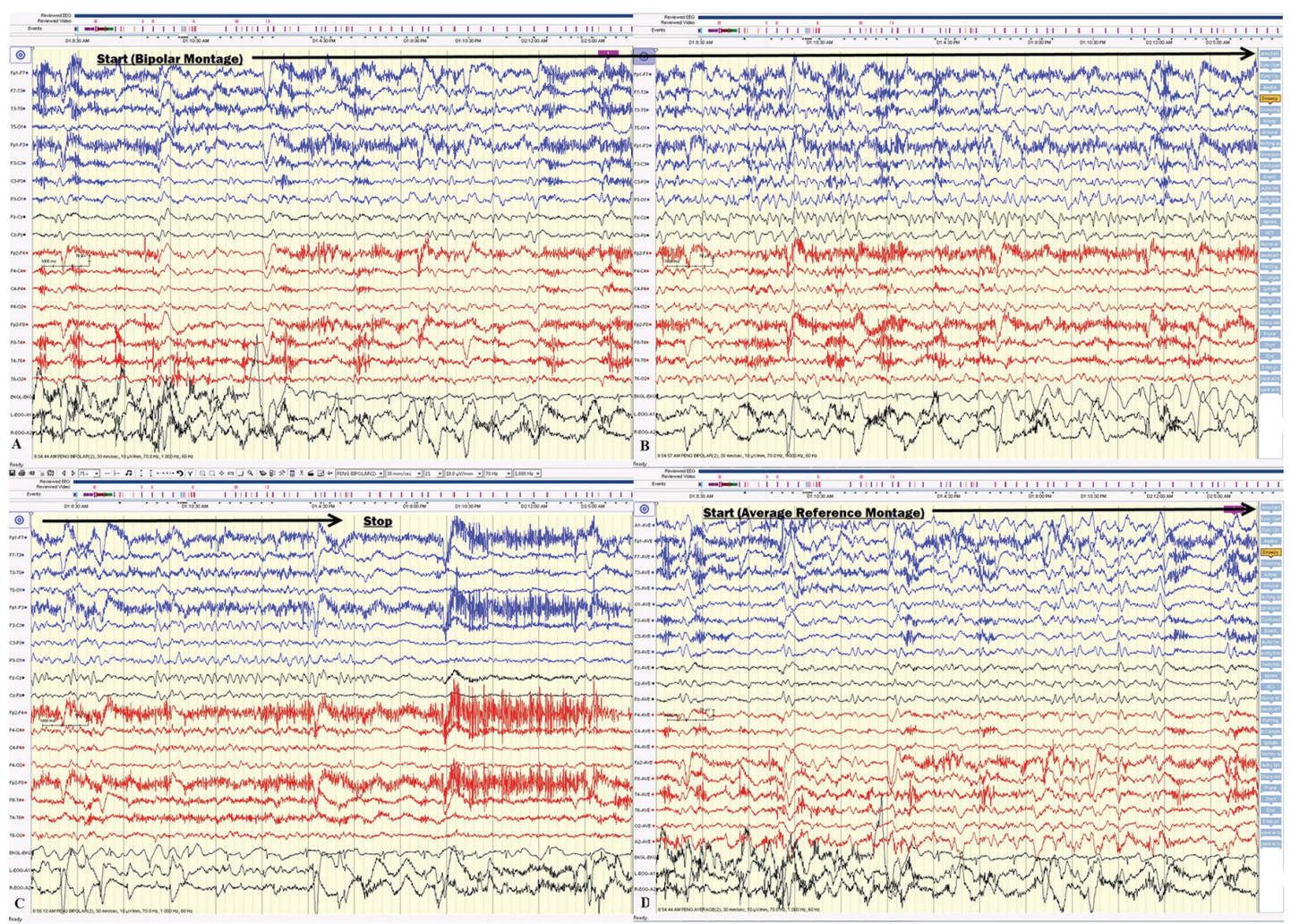

Figure 4. (A, B, C) The electrical seizures started with sudden appearance of repetitive epileptiform sharp waves over the left hemisphere displayed on a bipolar montage, which evolved in amplitude and frequency over the left hemisphere. (D) The onset of the same seizure displayed on an average reference montage.

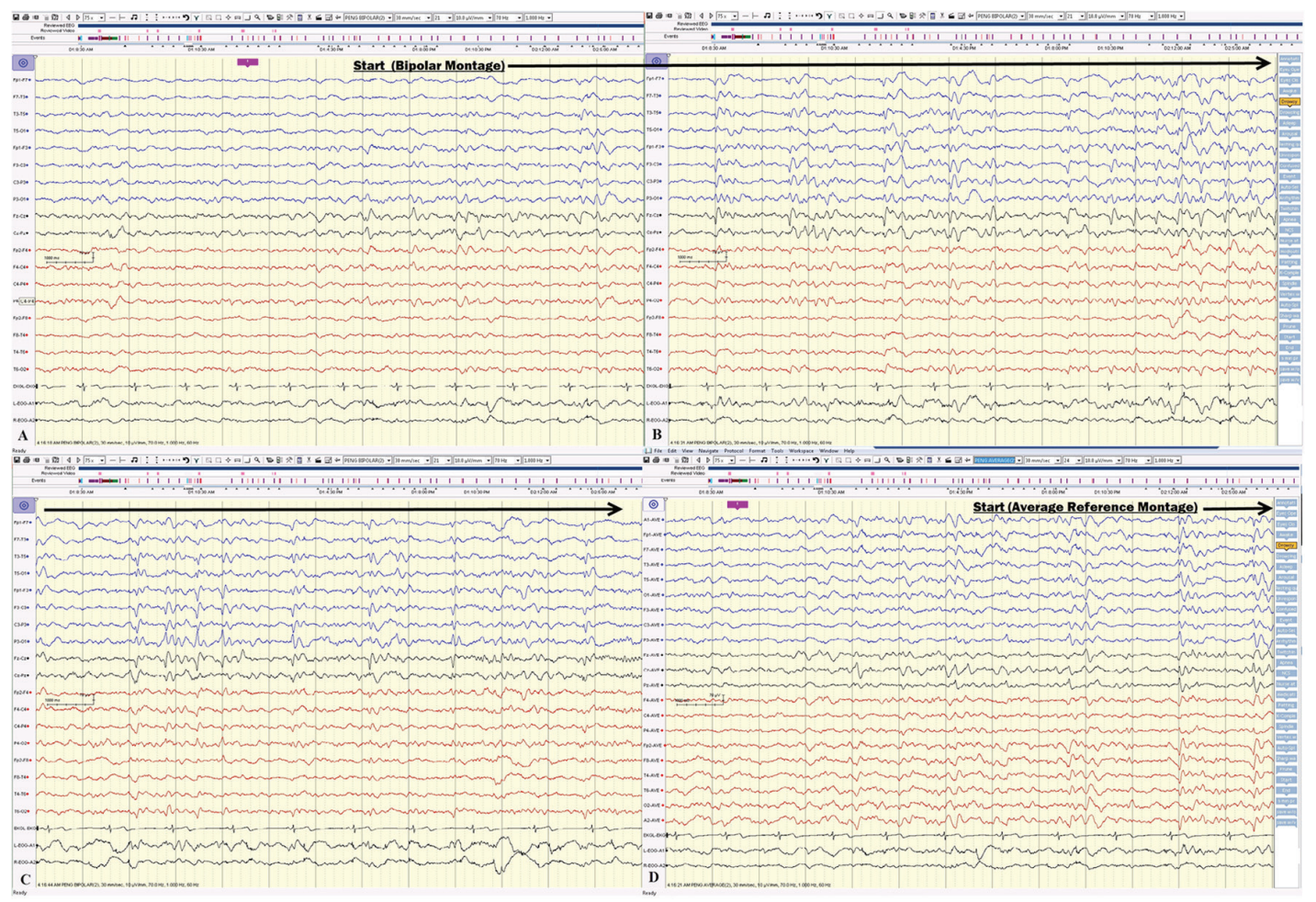

Figure 5. (A, B, C) Electrical seizure in the sleep. (D) The onset of the same seizure displayed on an average reference montage. 


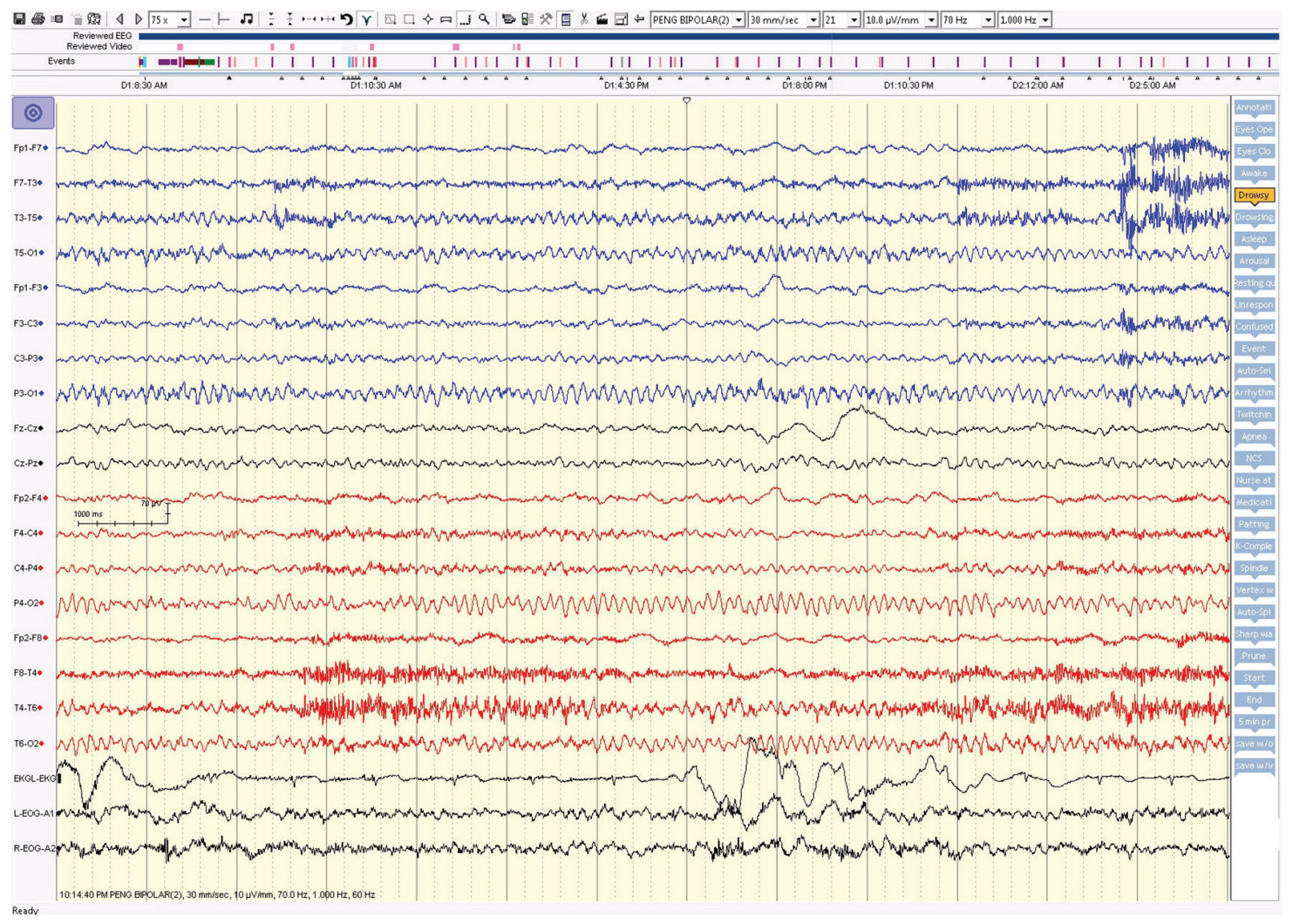

Figure 6. The awake EEG background was well preserved between seizures.

travenous solumedrol.

In this particular case, the MRI of the brain, EEG monitoring, and the clinical course suggested that the non-convulsive seizures, amnesia, the abnormal T2 signal changes of the bilateral hippocampus, amygdala, fornix and mammillary body in MRI of the brain were positively correlated.

\section{Discussion}

The case we present here is unique because the patient's fluctuating course allows us to reach a conclusion that non-convulsive seizures, amnesia, the abnormal T2 signal changes of bilateral hippocampus and fornix in MRI of the brain are correlated in this case.

This positive correlation supports the hypothesis that these non-convulsive seizure clusters are the primary cause of memory problems and MRI abnormalities. The MRI abnormalities of limbic encephalitis could be explained by post-ictal edema. The MRI abnormalities of reported cases of limbic encephalitis in the literature vary because the extent and severity of seizure vary.

The myriad of symptoms of limbic encephalitis can be explained by clusters of non-convulsing seizures [10]. The symptoms of limbic encephalitis vary because the extent and severity of non-convulsing seizure vary. This hypothesis predicts that patients with GAD antibodies may develop seizures from brain regions other than the limbic areas [2].
In this case, the temporal lobe seizure clusters are triggered by a systemic autoimmune disease process. Temporal lobes happen to be a vulnerable area of the brain in which seizures develop [10]. The non-convulsive seizure clusters could also potentially lead to hippocampal sclerosis and intractable seizures afterward [11].

High-dose intravenous solumedrol and aggressive antiseizure management are of help $[12,13]$. In this case, the majority of seizures were not convulsing, and intensive video EEG monitoring would be imperative to clarify the diagnosis and guide the treatment.

Aggressive seizure control in this stage potentially could modify the clinical course and prevent probable hippocampal sclerosis in the future [11].

The limitation of our hypothesis is that it comes from observation on one case.

\section{Conflicts of Interest}

All authors declare no conflict of interest.

\section{References}

1. Sharma A, Dubey D, Sawhney A, Janga K. GAD65 Positive Autoimmune Limbic Encephalitis: A Case Report and Review of Literature. J Clin Med Res. 2012;4(6):424- 


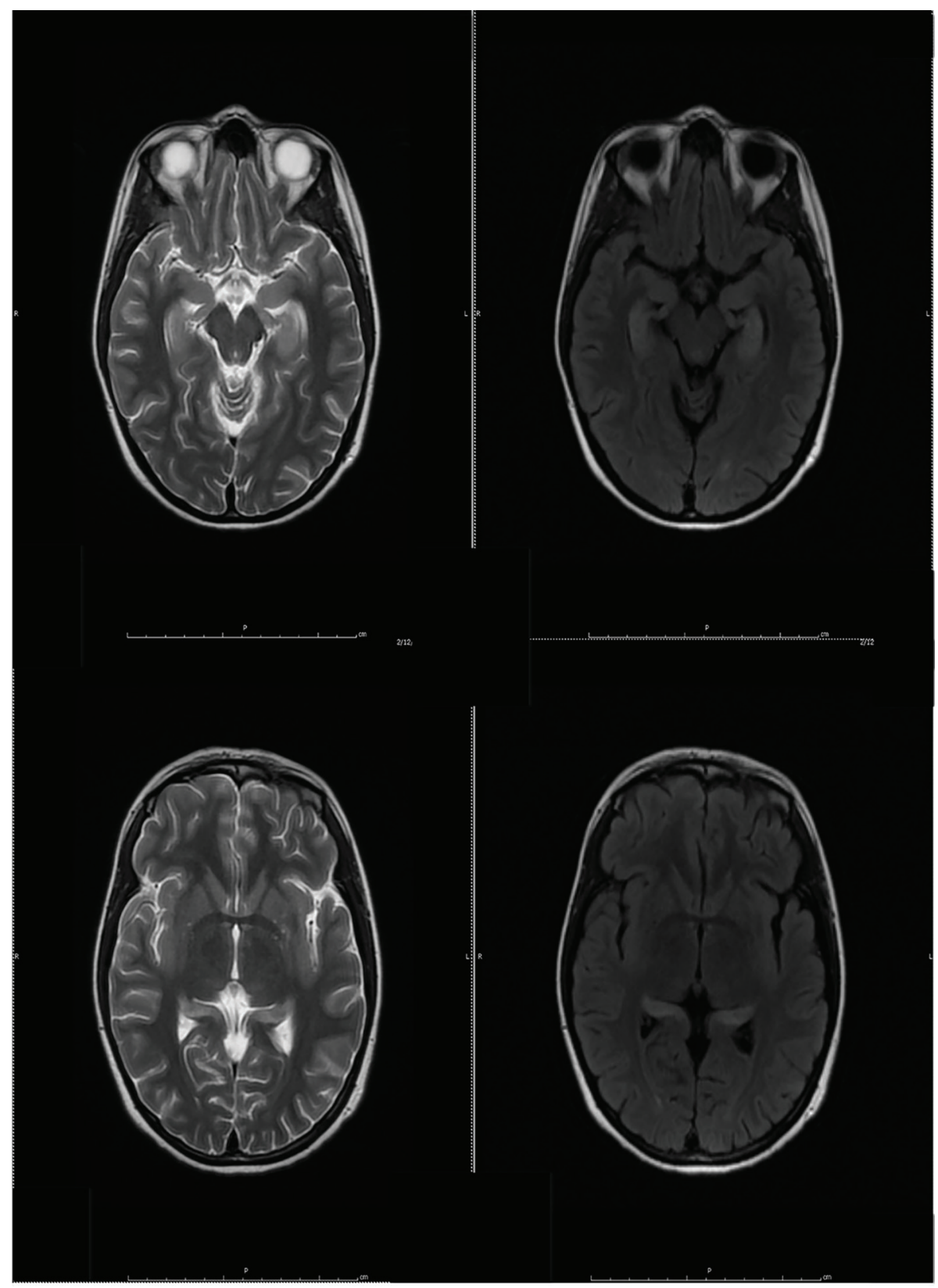

Figure 7. Follow-up MR examination showed significant interval decrease in the extent of T2 signal. MRI in Figure 3 and Figure 7 was 3 weeks apart.

428.

2. Kobayakawa Y, Tateishi T, Kawamura N, Doi H, Ohyagi Y, Kira J. [A case of immune-mediated encephalopathy showing refractory epilepsy and extensive brain MRI lesions associated with anti-glutamic acid decarboxylase antibody]. Rinsho Shinkeigaku. 2010;50(2):92-97.

3. Towns R, Pietropaolo M. GAD65 autoantibodies and its role as biomarker of Type 1 diabetes and Latent Autoimmune Diabetes in Adults (LADA). Drugs Future. 2011;36(11):847.

4. Vianello M, Tavolato B, Giometto B. Glutamic acid decarboxylase autoantibodies and neurological disorders.
Neurol Sci. 2002;23(4):145-151.

5. Treiman DM. GABAergic mechanisms in epilepsy. Epilepsia. 2001;42(Supp1 3):8-12.

6. Meldrum BS. GABAergic mechanisms in the pathogenesis and treatment of epilepsy. Br J Clin Pharmacol. 1989;27(Suppl 1):3S-11S.

7. Finelli PF. Autoimmune Limbic Encephalitis With GAD Antibodies. Neurohospitalist. 2011;1(4):178-181.

8. Arino $\mathrm{H}$, Hoftberger $\mathrm{R}$, Gresa-Arribas $\mathrm{N}$, MartinezHernandez E, Armangue T, Kruer MC, Arpa J, et al. Paraneoplastic Neurological Syndromes and Glutamic Acid Decarboxylase Antibodies. JAMA Neurol. 
2015;72(8):874-881.

9. Lin LY, Chang MH, Lee WJ. Paraneoplastic Limbic Encephalitis Associated with Adenocarcinoma of Lung. Acta Neurol Taiwan. 2014;23(3):108-112.

10. Bien CG, Elger CE. Limbic encephalitis: a cause of temporal lobe epilepsy with onset in adult life. Epilepsy Behav. 2007;10(4):529-538.

11. Thom M. Review: Hippocampal sclerosis in epilepsy: a neuropathology review. Neuropathol Appl Neurobiol.
2014;40(5):520-543.

12. Widman G, Golombeck K, Hautzel H, Gross CC, Quesada CM, Witt JA, Rota-Kops E, et al. Treating a GAD65 Antibody-Associated Limbic Encephalitis with Basiliximab: A Case Study. Front Neurol. 2015;6:167.

13. Malter MP, Frisch C, Zeitler H, Surges R, Urbach H, Helmstaedter C, Elger CE, et al. Treatment of immunemediated temporal lobe epilepsy with GAD antibodies. Seizure. 2015;30:57-63. 